Regards sur l'économie allemande

Bulletin économique du CIRAC

$69 \mid 2004$

Varia

\title{
Les entreprises allemandes à l'heure des normes IAS/IFRS
}

Caroline Guiot

\section{OpenEdition}

\section{Journals}

Édition électronique

URL : http://journals.openedition.org/rea/3323

DOI : $10.4000 /$ rea.3323

ISBN : 978-2-8218-0834-8

ISSN : 1965-0787

\section{Éditeur}

CIRAC

\section{Édition imprimée}

Date de publication : 1 décembre 2004

Pagination : 23-28

ISSN : 1156-8992

\section{Référence électronique}

Caroline Guiot, "Les entreprises allemandes à l'heure des normes IAS/IFRS », Regards sur l'économie allemande [En ligne], 69 | décembre 2004, mis en ligne le 27 janvier 2009, consulté le 19 avril 2019

URL : http://journals.openedition.org/rea/3323 ; DOI : 10.4000/rea.3323 


\section{Les entreprises allemandes à 1'heure des normes IAS/IFRS}

\section{Caroline Guiot}

Le $1^{\text {er }}$ janvier 2005, quelque 7000 sociétés européennes cotées devront établir leurs états financiers selon les normes comptables internationales (IAS: International Accounting Standards / IFRS : International Financial Reporting Standards). Alors que la presse économique française pointe du doigt le degré d'impréparation des entreprises françaises qui voient majoritairement ce basculement comptable comme une nouvelle contrainte bruxelloise, d'inspiration anglosaxonne de surcroît, les sociétés allemandes, elles, sont sereines, habituées depuis longtemps à des arrêtés comptables en plusieurs référentiels. Et elles pensent déjà à l'étape suivante : l'application des normes IAS/IFRS à toutes les entreprises, PME comprises. Pourtant, les changements sont importants : d'abord la conception de l'information financière est profondément bouleversée, ensuite, c'est l'organisation même des services internes des entreprises pour collecter les informations qui est à repenser... Soit un projet complexe, de longue haleine, à mener comme un challenge par les entreprises pour n'être pas pénalisées par les acteurs des marchés financiers qui, eux-mêmes, se préparent à ces changements de grande ampleur.

Le contexte de la globalisation des marchés financiers mondiaux appelait la création d'un référentiel comptable unique pour traduire les activités et les transactions des entreprises dans leurs états financiers: les normes comptables internationales ont été élaborées pour répondre à cette exigence. Et afin de créer les conditions nécessaires à la construction d'un marché européen des capitaux intégré et efficace dans ce contexte global, l'UE a adopté le 19 juillet 2002 un règlement sur l'application de ces normes. Celles-ci ont pour objectifs majeurs d'améliorer la comparabilité des entreprises à l'échelle internationale et d'assurer une plus grande transparence des données, notamment pour leurs utilisateurs : investisseurs (actionnaires), banques, analystes financiers, clients, fournisseurs... Or ces utilisateurs souhaitent pouvoir juger du patrimoine et des performances financières et non financières de l'entreprise à un instant donné.

Pour cela, les normes internationales fournissent un corps de principes permettant de comptabiliser toutes les opérations des entreprises, quel que soit leur secteur d'activité, et exigeant de fournir une somme importante d'informations en annexe. Cette nouvelle conception de l'information financière est à la fois plus globale et étendue. En effet, la transcription de la réalité économique nécessite une approche 'grand angle' : elle touche tous les éléments, financiers et non financiers, d'une entreprise, impliquant par là même tous ses services et divisions ; le champ des données à fournir s'élargit d'autant. Parallèlement, la forme des prescriptions est également modifiée. II ne s'agit plus seulement d'énoncer des règles comprenant l'énumération la plus exhaustive des cas possibles et leur traitement respectif, les normes IAS/IFRS sont volontairement un ensemble de principes dont il convient de saisir l'esprit, avant de les appliquer.

Dans l'esprit de ces normes, deux points majeurs modifient les approches comptables européennes (notamment celles de la France et de l'Allemagne) :

- le coût historique, c'est-à-dire le coût d'un bien à sa date d'acquisition, va être remplacé par la juste valeur, c'est-à-dire la valeur actuelle du bien, le prix auquel il serait

Intégration de l'UE dans le contexte mondial

Une conception élargie de l'information financière

Les principaux changements :

... juste valeur... 
... et substance économique

Exemples de traitement selon les normes IAS/IFRS

Toutes les fonctions de l'entreprise sont concernées

Un chantier long et coûteux... vendu ou remplacé à la date d'arrêté des comptes. Si ce principe permet de traduire la situation économique d'une entreprise à un instant donné, il est indéniable qu'il va entraîner une volatilité accrue des résultats, du fait de la fluctuation des cotations sur les marchés (matières premières, immobilier, titres financiers, etc.) des éléments ainsi comptabilisés ;

- la substance économique doit primer sur la forme juridique : il conviendra désormais de considérer les risques assumés et les avantages retirés par l'entreprise pour déterminer le traitement à adopter pour une transaction, sans tenir compte de montages juridiques qui pourraient masquer la réalité économique des relations entre l'entreprise et un tiers.

Parallèlement, l'accent est mis sur la transparence et l'information complète des utilisateurs des états financiers. C'est pourquoi certains sujets font l'objet d'annexes détaillées : information sectorielle, regroupement d'entreprise, rémunérations, etc.

Sans prétendre à une présentation exhaustive du contenu des normes IAS/ IFRS, voici quelques exemples de traitements comptables selon ces normes:

- Immobilisations corporelles : mise en place de la comptabilisation par composants, c'est-à-dire que chacun des éléments constituant un bien durable doit être identifié et comptabilisé séparément si sa durée d'utilisation est différente du bien dans sa totalité (remplacement plus fréquent de certaines pièces).

- Recherche et développement: les frais de R\&D ne sont plus automatiquement comptabilisés en charge, mais doivent être immobilisés (immobilisations incorporelles) à partir du moment où le produit passe en phase de développement, c'est-àdire quand la société a une " idée raisonnable » du succès de son produit.

- Locations : quelle que soit la forme juridique du contrat, seule l'analyse de la substance compte. Dans le cas d'un contrat de crédit-bail ou de location financière, celuici sera décomposé en actif et dette correspondante (comme si le bien avait été acquis au moyen d'un crédit bancaire classique) et inscrit comme tel au bilan.

- Chiffre d'affaires : le transfert des risques et des charges au client est le fait générateur de la comptabilisation d'un produit, et non plus la facturation.

- Avantages au personnel: toutes les formes de rémunérations et d'avantages au personnel doivent être comptabilisées, et actualisées (pour ce qui relève des charges futures, notamment les engagements de retraite).

- Périmètre de consolidation : l'application du principe de primauté de la substance économique sur la forme juridique revient à déterminer le périmètre de consolidation, non plus à travers un pourcentage de détention d'actions, mais en fonction de l'analyse du contrôle et de l'influence d'une société sur une autre, sans s'arrêter aux montages juridiques.

- Goodwill : l'amortissement des «survaleurs » n'est plus autorisé. L'écart entre le coût d'acquisition et la juste valeur des actifs et passifs d'une société reprise ne sera plus amorti ; il conviendra de recalculer sa valeur recouvrable à chaque clôture à l'aide de tests de dépréciation.

Ces quelques exemples illustrent parfaitement la complexité de la mise en œuvre des normes IAS/IFRS. Car celle-ci ne touche pas uniquement le domaine comptable. Toutes les fonctions de l'entreprise sont concernées, dans le but de collecter les informations pertinentes pour l'établissement des états financiers: les informaticiens pour adapter le paramétrage des logiciels, les commerciaux pour relever la date d'acception du bien par le client (comptabilisation du revenu), les juristes pour valider les clauses des contrats, les responsables de production pour identifier les composants des immobilisations, les chercheurs pour déterminer le passage de la phase de recherche à la phase de développement d'un produit, les responsables des ressources humaines pour recenser l'ensemble des avantages au personnel (actuels ou futurs), et ainsi de suite. Ce sont ainsi de nombreux opérationnels qui n'ont jamais été directement sollicités, et qu'il faut former pour répondre à cette exigence de remontée des informations jusqu'aux services comptables qui les enregistrent.

II n'est donc pas étonnant de constater, à travers une enquête menée par le cabinet Mazars en novembre 2003, que 43 \% des entreprises cotées européennes perçoivent le passage aux normes IAS/IFRS comme un chantier long et coûteux. Le projet de conversion est généralement décomposé en trois ou 
quatre phases : (1) diagnostic - planification du projet et affectation des ressources, recensement des divergences entre les normes appliquées par le groupe et les normes IAS/IFRS; (2) préparation - définition et validation des traitements comptables retenus, études d'impacts sur les états financiers et recensement des données et informations supplémentaires nécessaires, mise en place d'un plan de formation ; (3) basculement - déploiement du plan de conversion des données et validation post-conversion, mise à jour des manuels de procédures, tenue des comptes et préparation des états financiers ; (4) suivi outre celles qui ne sont pas définitives, les normes IAS/IFRS ne sont pas figées et évoluent régulièrement, d'où un suivi nécessaire.

\section{Trente ans de travaux pour des normes comptables harmonisées}

Ce n'était au départ qu'un idéal des années 1970 : pouvoir comparer les résultats des entreprises du monde entier traduits dans une même comptabilité... De cette idée est né l'International Accounting Standards Committee (IASC), fondé le 29 juin 1973 à Londres, avec pour objectif, si ce n'est d'harmoniser toutes les comptabilités mondiales, du moins d'en créer une commune, d'envergure internationale, ce qui n'était pas moins ambitieux. Rappelons que cette organisation est totalement indépendante des Etats qui y participent, que les membres ont des origines professionnelles diverses (anciens auditeurs, universitaires, analystes financiers), sont volontaires, à temps partiel et initialement non rémunérés.

Quelques dates clés de l'action de l'IASC :

- 1973 : inauguration de l'IASC, dont les membres fondateurs sont l'Allemagne, l'Australie, le Canada, les Etats-Unis, la France, le Japon, le Mexique, les Pays-Bas et le Royaume-Uni, réunis dans le Board de l'IASC.

- 1974 : publication du premier projet de norme (« Exposure Draft ») : IAS 1 - Présentation des règles comptables.

- 1977 : fondation de I'IFAC : International Federation of Accountants, regroupant les professionnels des métiers de la comptabilité et travaillant en étroite collaboration avec l'IASC.

- Années 1980 : plusieurs pays intègrent le Board. Les travaux et les consultations s'organisent.

- 1986 : les analystes financiers rejoignent le Board.

1988 : le FASB (normalisateur américain) rejoint le Board, en tant qu'observateur.

- 1990 : la Commission Européenne rejoint le Board, en tant qu'observateur.

- Années 1990 : publications de plusieurs normes IAS.

- 1997 : création du SIC : Standing Interpretations Committee, comité chargé d'interpréter les normes dans leurs applications.

- 2001 : réorganisation de I'IASC, qui devient IASB (Board), dont le président est Sir David Tweedie. L'indépendance financière est assurée par une fondation (IASC Foundation).

- 2002 : La publication des normes révisées en vue de leur application s'accélère, et l'UE adopte des règlements pour l'application des IFRS : le règlement $n^{\circ} 1606 / 2002$ du Parlement et du Conseil européens, relatif à l'application des normes comptables internationales, adopté le 19 juillet 2002, ainsi que les règlements $n^{\circ} 1725 / 2003$ et $n^{\circ} 707 / 2004$ de la Commission.

Source des données : IASB (www.iasb.org)

Les experts s'accordent à estimer la durée du projet de passage aux normes IAS/IFRS à un minimum de douze mois. D'après un sondage réalisé par le cabinet PricewaterhouseCoopers en mars 2004, plus d'un tiers des groupes français $(37 \%)$ estime être en retard dans le projet de passage aux normes IAS/ IFRS, et $59 \%$ estiment être dans les temps. Paradoxalement, dans l'enquête européenne menée par Mazars quatre mois plus tôt, près de $90 \%$ des sociétés cotées allemandes avaient dépassé la phase de diagnostic. Cette avance s'explique en partie par l'autorisation précoce (octobre 2001) donnée par le code de commerce allemand de publier des états financiers consolidés selon les normes IAS/IFRS.

C'est une période de transition entre l'ancien référentiel national et le nouveau référentiel international qui s'ouvre. Et si l'objectif des normes IAS/IFRS est une plus grande transparence et une meilleure comparabilité des résultats et des performances des entreprises, à court terme, cela risque d'être fort complexe, car toutes les sociétés n'en sont pas aux mêmes phases. Le management des sociétés va donc devoir faire preuve de pédagogie pour expliquer l'avancement de leur projet de conversion aux acteurs des marchés financiers. Compte tenu de l'enjeu, les entreprises sont d'ailleurs épaulées par plusieurs institutions : les cabinets d'expertise comptable et de commissariat aux comptes sont en pre-
La France en retard, l'Allemagne sereine

Période de transition délicate et nécessaire pédagogie 
Quelques outils pour aider les entreprises

1008 sociétés concernées en Allemagne

Quid des autres entreprises ?

Les différents référentiels comptables vont coexister mière ligne pour fournir prestations et outils techniques, mais aussi formations adaptées aux spécificités des secteurs des entreprises. Les grandes banques et autres autorités de régulation des marchés financiers proposent plutôt des conseils pour aider les entreprises à communiquer sur les changements affectant les états financiers lors du basculement.

Ainsi par exemple, pour rendre possible la comparaison entre l'avant et l'après conversion, le Comité européen des régulateurs des marchés de valeurs mobilières (CESR) a diffusé un plan en quatre étapes :

1. lors de la publication des états financiers 2003, décrire les mesures engagées pour assurer le succès du projet de transition vers les normes IAS/IFRS, et le degré d'avancement ;

2. lors de la publication des états financiers 2004, fournir une information pertinente sur les impacts du nouveau référentiel sur les états financiers ;

3. la publication des comptes intermédiaires 2005 devra avoir été préparée dans le respect des normes IAS/IFRS ;

4. Iors de la publication des états financiers 2005, l'information comparative devra être accessible et claire.

Et afin de faciliter encore l'élaboration des états financiers comparés, à l'issue de la période de transition, un format indicatif de présentation (valable pour le bilan, le compte de résultat, le tableau de variation des capitaux propres et le tableau des flux de trésorerie) est fourni par le CESR :

\begin{tabular}{|l|c|c|c|c|}
\hline $\begin{array}{c}\text { Eléments des } \\
\text { états financiers }\end{array}$ & $\begin{array}{c}\text { Exercice 2005 } \\
\text { en normes } \\
\text { IAS/IFRS }\end{array}$ & $\begin{array}{c}\text { Exercice 2004 } \\
\text { en normes } \\
\text { IAS/IFRS } \\
\text { (retraité) }\end{array}$ & $\begin{array}{c}\text { Exercice 2004 } \\
\text { en ancien } \\
\text { référentiel } \\
\text { (tel que publié) }\end{array}$ & $\begin{array}{c}\text { Exercice 2003 } \\
\text { en ancien } \\
\text { référentiel } \\
\text { (tel que publié) }\end{array}$ \\
\hline
\end{tabular}

Source des données : AMF, 03/2004 (www.amf-france.org)

Au $1^{\text {er }}$ janvier 2005, environ 7000 sociétés de l'UE devront donc publier leurs comptes consolidés selon les normes IAS/IFRS. Le règlement n¹606/2002 du Parlement et du Conseil européens, relatif à l'application des normes comptables internationales, stipule que ce sont les sociétés immatriculées dans l'un des 25 Etats membres de l'UE et dont les titres (actions ou obligations) sont cotés sur un marché réglementé européen. En RFA, plus d'un millier de sociétés répondant à ces critères a été recensé : 791 dans le secteur de l'industrie, du commerce et des services, et 217 établissements financiers et d'assurance.

Or ces 1008 sociétés cotées en Allemagne ne représentent qu'une partie infime du tissu économique national, composé à $99 \%$ de PME, le plus souvent au statut de sociétés de personnes ou en nom personnel. Or dans l'industrie, la forme juridique qui domine avec près de $40 \%$ est celle de la $\mathrm{GmbH}$, équivalente à la SARL - avec un capital fermé et une équipe dirigeante familiale. L'IASB travaille d'ailleurs à un système 'allégé' de normes pour les PME et a ouvert une concertation en ce sens, à laquelle participent activement la fédération allemande de l'industrie BDI et celle des chambres du commerce et de l'industrie DIHK, fidèles en cela à leur rôle de défenseur des intérêts de leur branche ou de leurs membres, en Allemagne comme dans les enceintes bruxelloises. En l'état actuel, aucune prescription n'a été publiée. En revanche, le règlement européen laisse la liberté au législateur national d'autoriser ou d'obliger les sociétés non cotées à établir leurs états financiers en normes IAS/ IFRS. La France et l'Allemagne ont choisi d'autoriser l'adoption volontaire des normes internationales par les entreprises.

Dans ces conditions, les normes comptables internationales vont coexister avec les référentiels nationaux. D'une part parce que les comptes individuels de toutes les entreprises, cotées ou non, devront être établis selon le référentiel local (Handelsgesetzbuch, HGB, en Allemagne). D'autre part, parce que les distributions de dividendes et la fiscalité s'appuient sur les comptes en référentiel national. Ce qui pose à terme la question de l'harmonisation des régimes fiscaux dans le cadre d'une unification des normes comptables... 
Mais quel serait donc l'avantage, pour une société qui n'y serait pas obligée par la loi, de produire des états financiers selon les normes comptables internationales ? Si une transition est, à l'évidence, particulièrement coûteuse pour la plupart des entreprises de taille petite et moyenne, elle n'en présente pas moins plusieurs avantages, notamment dans ses rapports avec ses interlocuteurs économiques, financiers et institutionnels.

Pour bien comprendre les enjeux, rappelons que le Mittelstand représente, seIon la définition allemande, quelque 3,3 millions d'entreprises : $40 \%$ oeuvrent dans le secteur des services, $22 \%$ dans le commerce (essentiellement des entreprises artisanales) et $37 \%$ dans le secteur industriel. Le Mittelstand est inégalement internationalisé : alors que les entreprises de services et du commerce sont principalement centrées sur le marché domestique, les $4 / 5^{\mathrm{e}}$ des entreprises du secteur des biens manufacturés se positionnent à l'export (la branche de la machine-outil est même leader mondial). Dans le contexte de la globalisation des marchés et de l'intensification de la concurrence, y compris sur le marché communautaire, il est indéniable que la publication des comptes dans un référentiel international est stratégique. En effet, en renforçant la lisibilité de leurs résultats et de leurs performances, ces PME accroissent leur compétitivité et leur croissance potentielles.

Par ailleurs, une autre caractéristique importante du Mittelstand allemand est son fonctionnement en réseau : la sous-traitance industrielle, le service aux entreprises, l'omniprésence des banques, en tant que financeurs, actionnaires et administrateurs, le lien tissé au travers des fédérations professionnelles, tout concourt à une forte intégration des PME allemandes. Et quand bien même elles ne sont actives que sur le marché domestique, leurs liens de coopération étroits avec des sociétés cotées, elles, fortement internationalisées, les poussent à mettre en œuvre les normes comptables internationales. Comme l'explique Sven Hayn, associé du cabinet Ernst \& Young, « tant l'alliance stratégique traditionnelle entre clients et fournisseurs que les nouvelles relations $B 2 B$ et B2C nécessitent un langage comptable commun, et l'Union européenne a décidé que ce serait les normes IAS/IFRS ». Le langage commun donnera donc à ces entreprises l'avantage compétitif d'une nouvelle transparence, contribuant ainsi indirectement à la mise en œuvre des objectifs de la Stratégie de Lisbonne. C'est dès lors un effet d'entraînement qui conduira les entreprises, en partant des sociétés cotées jusqu'aux PME, à adopter les normes IAS/IFRS.

De plus, la conversion volontaire aux normes comptables internationales pourrait se révéler payante vis-à-vis des acteurs sur les marchés financiers. Une 'prime d'information financière' pourrait par exemple récompenser une telle attitude : meilleur rating des analystes financiers, meilleurs taux d'intérêt de la part des banques... Dans le cas contraire, la non application des normes IAS/IFRS pourrait être considérée comme de l'obstruction à l'accès à l'information, et sanctionnée comme telle. C'est ainsi que la conversion pourrait devenir une obligation de fait, sous la pression des acteurs des marchés financiers, actionnaires, banques, analystes, etc. De ce point de vue, Sven Hayn estime que les trois quarts des entreprises n'ont pas encore réalisé le lien entre la publication de leurs comptes selon les normes IAS/IFRS et la possibilité d'améliorer leurs opportunités de financement. Et de rappeler que les normes comptables internationales ont été élaborées pour répondre aux besoins des investisseurs, principaux utilisateurs des états financiers. En outre, dans le cas du Mittelstand, ces investisseurs sont en premier lieu les banques, qui, en quête d'une meilleure lisibilité des activités des PME dans le cadre de leur propre modernisation (« Bâle II »), poussent de leur côté à une standardisation des bilans.

Quoi qu'il en soit, la question de l'adoption des normes IAS/IFRS par le Mittelstand donne lieu à un vif débat outre-Rhin, et ce débat se déroule largement sur la place publique. Ainsi, le quotidien Frankfurter Allgemeine Zeitung a ouvert à l'automne ses pages aux réflexions des experts. Si certains estiment que la
La conversion volontaire, un avantage compétitif...

... sur le marché international

Le fonctionnement en réseau aura un effet d'entraînement

Une prime à l'information financière

Mais le débat reste vif 
surcharge de travail que représenterait une telle adoption serait disproportionnée et plaident un statu quo, d'autres vont jusqu'à préconiser une réforme du droit allemand des sociétés ; d'autres encore estiment qu'une conversion s'impose de fait, puisqu'elle n'est qu'une manifestation parmi d'autres du processus d'intégration européenne des marchés. La fédération DIHT, pour sa part, mène un travail d'explication pédagogique auprès de ses adhérents, via sa plateforme Internet (www.diht.de) et ses activités internes d'information/formation. Ainsi, d'une manière plus générale, la question des normes comptables contribue aussi à alimenter la réflexion sur la modernisation des structures juridiques et capitalistiques du Mittelstand. Impulser aux PME la volonté du changement, ne serait-ce qu'au plan comptable, revient en ce sens à soutenir leur compétitivité future dans un environnement réglementaire en pleine mutation - fidèlement à l'esprit des objectifs définis lors du Sommet européen de Lisbonne en 2000.

LA MISE EN CEUVRE des nouvelles normes comptables internationales a contribué à enclencher un processus de modernisation du tissu économique allemand. Loin de voir cette conversion comme une contrainte bruxelloise, les sociétés cotées l'exploitent comme une opportunité vis-à-vis des marchés : amélioration des procédures internes et plus grande transparence mettent en exergue les adaptations dont les entreprises allemandes sont capables pour répondre aux exigences des acteurs. Sous l'action de l'harmonisation en cours des normes régissant les activités bancaires et boursières, la modernisation se diffuse de proche en proche jusque dans le secteur des PME, favorisée par l'ouverture internationale d'un Mittelstand industriel allemand qui dispose par ailleurs de multiples relais d'information et d'incitation du fait du fonctionnement en réseaux de partenariats comme de représentations propre à l'économie allemande. Les plus internationalisées des PME, rompues à la diversité des normes comptables, s'accommodent de la coexistence des normes IAS/IFRS et des règles nationales, se préparant pour l'heure à une double présentation de leur bilan. Quant aux autres qui, tributaires du seul marché domestique, se contenteront d'appliquer les normes nationales, elles finiront forcément par maîtriser elles aussi les différents langages comptables dès lors qu'elles cultivent des liens de partenariat étroit avec des PME plus internationalisées. Or, conjugué à l'adoption du rating et à la découverte de nouveaux instruments financiers, cet apprentissage aura pour effet de moderniser durablement une culture entrepreneuriale encore souvent enfermée dans le cercle familial. A l'avenir plus transparente, l'économie allemande y gagnera aussi en attractivité pour l'investissement.

\section{Indications bibliographiques}

Bourgeols I., " Comment les PME allemandes passent-elles au numérique? », in Dossier Stratégie de Lisbonne, Regards sur l'économie allemande, 61/03

BDI, Zielmarke Lissabon: Herausforderungen für den industriellen Mittelstand in einem erweiterten Europa. Strategien und Positionen für eine europäische Mittelstandspolitik 2005-2010, 11-10-2004

BDI / DIHT, Stellungsnahme zum Diskussionspapier des IASB zur Entwicklung von internationalen Rechnungslegungsstandards für kleine und mittelgroße Unternehmen, 09/04

ERNST \& Young, Viele Unternehmen unterschätzen Auswirkungen der IFRS-Umstellung, $12 / 03$

"IFRS - Révolution dans l'entreprise ", dossier publié par Les Echos en partenariat avec PricewaterhouseCoopers, 13-05-2004

MAZARS, Les impacts du passage aux normes IAS-IFRS sur les entreprises européennes : entre opportunités et complexités, 11/2003 (www.mazars.com)

Mind 03 - Mittelstand in Deutschland, Impulse / Institut für Mittelstandsforschung Bonn (ifm-bonn.de)

PricewaterhouseCoopers / PwC Deutsche ReVision, IAS/IFRS - Kapitalmarktorientierte Unternehmen in Deutschland, 04/2004 